\title{
BMJ Open Non-inferiority and cost-effectiveness trial of isolated biceps tenotomy versus tenotomy with rotator cuff repair in patients with stage 2-3 Goutallier fatty degenerative cuff lesions (TenCuRe study): protocol of a multicentre randomised controlled trial
}

\author{
Freek Hollman, Nienke Wolterbeek (D) , Gie Auw Yang
}

To cite: Hollman $\mathrm{F}$, Wolterbeek N, Auw Yang G. Non-inferiority and costeffectiveness trial of isolated biceps tenotomy versus tenotomy with rotator cuff repair in patients with stage 2-3 Goutallier fatty degenerative cuff lesions (TenCuRe study): protocol of a multicentre randomised controlled trial. BMJ Open 2020;10:e032936. doi:10.1136/ bmjopen-2019-032936

- Prepublication history for this paper is available online. To view these files, please visit the journal online (http://dx.doi org/10.1136/bmjopen-2019032936).

Received 12 July 2019 Revised 04 December 2019 Accepted 07 January 2020

Check for updates

(C) Author(s) (or their employer(s)) 2020. Re-use permitted under CC BY-NC. No commercial re-use. See rights and permissions. Published by BMJ.

Orthopedic Surgery, St. Antonius Ziekenhuis, Nieuwegein, The Netherlands

Correspondence to Dr Nienke Wolterbeek; n.wolterbeek@ antoniusziekenhuis.nl

\begin{abstract}
Introduction For patients who are diagnosed with lesions of the rotator cuff that present advanced levels of fatty degeneration, arthroscopic repair of the rotator cuff remains controversial. This controversy can be attributed to the frequently reported high failure rate of the tendon fixation and the fact that it remains unclear why repair for these tears results in significant clinical improvement independent of the occurrence of such a re-tear.

Recent publications have reported comparable clinical improvements when merely a tenotomy of the long head of the biceps tendon was performed and the rotator cuff tear was left untreated. These observations raise questions on the value of performing the more extensive cuff repairs in degenerative cuff tears. Even more, rehabilitation after an isolated tenotomy is much less cumbersome as compared with rehabilitation after rotator cuff repair and, therefore, might result in improved patient satisfaction. The goal of this trial is to study function and quality-of-life of patients undergoing arthroscopic biceps tenotomy with or without an additional cuff repair and to include an economic evaluation.
\end{abstract}

Methods and analysis This multicentre randomised controlled non-inferiority trial, including an economic evaluation, is designed to compare the short-term and long-term outcome of patients who underwent an arthroscopic tenotomy of the long head of the biceps tendon with or without a cuff repair. We will include 172 patients with stage 2-3 Goutallier fatty infiltration cuff tears and with clinical symptoms of biceps pathology. Primary outcome is the rotator cuff specific quality-of-life (Western Ontario Rotator Cuff index) on the short term (6 months postoperatively). Secondary outcomes are quality-of-life 1, 2 and 5 year postoperatively and function (Constant-Murley score, glenohumeral range of motion), recovery status, pain (visual analogue scale), economic evaluation, satisfaction of treatment on the short-term and long-term and re-tear rate at 6 months determined with an ultrasound.
Strengths and limitations of this study

- This will be the first randomised controlled trial to study the function and quality-of-life of patients undergoing arthroscopic biceps tenotomy with or without an additional cuff repair.

- The study is carefully designed including adequate sample size, permuted block randomisation stratified for centre, blinded analyses and prospective trial registration to reduce selection and confounding bias.

- Due to the obvious differences in postoperative rehabilitation protocols, patients, surgeons and researchers will not be blinded for treatment.

Ethics and dissemination This trial has been approved by the Medical Research Ethics Committees United (MEC-U), Nieuwegein, the Netherlands (NL54313.100.15) and will be performed in accordance with the Declaration of Helsinki with the Medical Research Involving Human Subjects Act (WM0). The results of this study will be reported in peer-reviewed journals and at (inter)national conferences. Furthermore, we will share our findings with the appropriate guideline committees.

Trial registration number The Dutch Trial Registry (NL4010).

\section{INTRODUCTION}

Rotator cuff pathology is a common condition which prevalence increases with age. ${ }^{1-3}$ The aetiology, incidence and the clinical significance of fatty degeneration of the rotator cuff muscles are still relatively unknown due to the lack of reliable measurements. Tendon quality can be assessed on MRI by the level of fatty infiltration, atrophy and retraction. In most studies the rotator cuff tear is defined 
irreparable if fatty infiltration $\geq$ stage 3 Goutallier, ${ }^{4}$ the tendon is retracted to the height of the glenoid (Thomazeau stage $3-4$, on the coronal plane ${ }^{5}$ ) and the acromiohumeral interval (AHI) is less than $7 \mathrm{~mm} \cdot{ }^{6-8}$ Furthermore, the severity of cuff arthropathy and the motivation to comply with the intensive rehabilitation programme after arthroscopic cuff repair (ACR) determines whether a cuff repair or other surgical treatment such as arthroplasty or superior capsular reconstruction is indicated. ${ }^{59}$

Among several identified negative prognostic factors for healing, fatty infiltration is frequently described as a predictor for healing of the rotator cuff. Healing implies anatomic integrity on the footprint after full recovery from surgery. Several clinical studies described the functional and radiographic outcome after ACR for the level of fatty infiltration. ${ }^{410-17}$ Fatty infiltration stages $0-1$ Goutallier (0\%-25\% fatty infiltration) have good to excellent clinical outcomes. Among patients with fatty infiltration stage 4 Goutallier ( $>75 \%$ fatty infiltration) the clinical outcome after ACR is poor with high failure rates and minor functional improvement. ${ }^{10}$ Within the remaining group, fatty infiltration stage 2-3 Goutallier, it is still under discussion whether cuff repair is indicated, mainly caused by the high re-tear rate. However, despite this high re-tear rate, patients seem to improve significantly with only minor functional difference compared with healed repairs. ${ }^{11-13}$

The biceps tendon is often involved in the presence of cuff arthropathy, especially in shoulders with stage 2-4 Goutallier. ${ }^{18}$ Therefore, in this patient group, biceps tenotomy or tenodesis could be considered as an alternative treatment instead of a cuff repair. Results of isolated tenotomy or tenodesis are comparable to the long-term results of cuff repair in this specific patient group. ${ }^{6-8}$ In addition, patients with a rotator cuff tear and good quality tendons for which an ACR and additional biceps tenotomy or tenodesis was performed also show comparable results. ${ }^{11}{ }^{19-23}$ Furthermore, there are several cases described in which a spontaneous ruptured long head of the biceps tendon (LHBT) from its insertion, in shoulders with a conservatively treated rotator cuff tear, has led to a significantly relieve of pain and improvements on function. ${ }^{24}$ These observations raise questions on the value of performing the more extensive and therefore expensive cuff repairs in degenerative cuff tears.

Rehabilitation after an isolated tenotomy is much less cumbersome compared with rehabilitation after rotator cuff repair. Patients undergoing an isolated biceps tenotomy might be able to resume work and daily activities much faster after surgery as their shoulders are directly functional without any restrictions. The patients who undergo a rotator cuff repair will be immobilised for 6 weeks in an anti-rotation sling and will need an additional 3-6 months for functional recovery, which will lead to more productivity losses. Therefore, the goal of this non-inferiority trial is to study the short-term function and quality-of-life in patients not responding to conservative therapy undergoing arthroscopic biceps tenotomy with or without an additional cuff repair and to include an economic evaluation.

We hypothesise that patients who undergo an isolated tenotomy of the LHBT in the presence of a degenerative rotator cuff tear, stage 2-3 Goutallier fatty infiltration, will have non-inferior functional results and quality-oflife 3, 6 and 12 months after surgery as compared with patients undergoing tenotomy combined with a rotator cuff repair. At 1 year after surgery we assume reduced costs with isolated tenotomy treatment.

\section{METHODS AND ANALYSIS \\ Study design}

This study is a multicentre randomised controlled noninferiority trial, including an economic evaluation. Prior to the start of inclusion, the trial was registered at the Dutch Trial Registry.

\section{Setting}

Patients are recruited at the orthopaedic outpatient clinics of the participating medical centres. Recruitment started in six Dutch hospitals. To ensure recruitment, additional hospitals will be added to the trial. Eligible participants are randomised into two equal groups receiving either arthroscopic biceps tenotomy with an additional cuff repair or arthroscopic biceps tenotomy without an additional cuff repair at the hospital of inclusion. The first patient was included on 22 October 2018. It is estimated that the inclusion period will be 3 years. Taken into account the 5 years follow-up period, the total study duration will be 8 years.

\section{Study population}

Patients $\geq 60$ years of age with MRI-confirmed symptomatic, stage 2-3 Goutallier fatty infiltration(FI) rotator cuff tears will be recruited. Other inclusion criteria are:

Preoperative inclusion criteria:

- History of shoulder complaints $>6$ months suggestive for a degenerative rotator cuff with or without nonsignificant preceding trauma.

- Cuff lesion, involving the supraspinatus and or infraspinatus tendon, with at least one of the involved tendons having stage 2-3 Goutallier fatty infiltration.

- Not responding to conservative treatment (eg, physiotherapy, corticosteroid injection).

- Willing and able to comply with the study protocol.

- Sufficient understanding of the Dutch language.

Perioperative inclusion criteria:

- Perioperative pathologic involvement of the LHBT including: (minor signs of) degeneration, or signs of inflammation, or instability (subluxation or dislocation), or partial tears or lesions of the superior labrum anterior and posterior (SLAP).

Patients will be excluded if one or more of the following exclusion criteria are met:

Preoperative exclusion criteria: 
- Cuff arthropathy according Hamada classification $\geq$ grade $3 .^{9}$

- Pseudoparalysis (active elevation limited to $45^{\circ}$ ).

- Full subscapularis tendon tear (delamination or partial avulsion accepted).

- Injury of the teres minor tendon.

- Mainly complaints from an osteoarthritis acromioclavicular joint and less from a subacromial origin, determined clinically or by subacromial/acromioclavicular infiltration.

- Shoulder instability for which labral repair is indicated.

- Irreparable rotator cuff tear, based on MRI (fatty infiltration Goutallier stage 4, retraction on the coronal plane to the level of the glenoid, severe atrophy).

- Unsuccessful surgery in the affected shoulder in history or surgery in the affected shoulder $<1$ year ago.

- Ipsilateral neurological pathology possibly affecting functional outcome.

- Full tear of the biceps tendon.

- Frozen shoulder.

- Cervical spine pathology affecting the functional outcome.

- Body Mass Index (BMI) $>35 \mathrm{~kg} / \mathrm{m}^{2}$.

- Time between surgery and MRI $>6$ months.

- Fracture of the humeral head involved in the cuff tear. Perioperative exclusion criteria:

- Perioperative fully healthy macroscopic aspect of the LHBT.

- Irreparable rotator cuff tear, based on perioperative findings.

\section{Recruitment and consent}

At the initial presentation, when it is suspected that the patient might be eligible, the patient will be verbally informed about the study and will receive the studyspecific information letter. As part of standard care, not as part of this study, an MRI will be conducted to confirm the diagnosis of a rotator cuff tear and for establishing a treatment plan. During the second visit (approximately 2 weeks later), when the results of the MRI are discussed, it will be established whether the patient is actually eligible. If eligible, the study protocol is explained in detail and informed consent is signed if patient is willing to participate. Participants are given a copy of the informed consent form and are informed that they can withdraw at any time during the study.

\section{Randomisation and blinding}

Randomisation is performed in a 1:1 ratio by a computerised software programme (REDCap) ${ }^{25}$ using random permuted blocks with block size 8 , stratified for centre. After informed consent is signed, patients will be entered in the online randomisation programme by the orthopaedic surgeon or study coordinator and are assigned to either arthroscopic biceps tenotomy with or without an additional cuff repair. Due to the obvious differences in postoperative rehabilitation protocols, patients, surgeons and researchers will not be blinded for treatment. Statistical analysis will be performed blinded.

\section{Treatment groups}

The procedures are only performed by orthopaedic surgeons experienced in arthroscopic rotator cuff surgery. Surgical techniques, such as patient positioning, portal placement and the use of assistive instruments such as knives, forceps, shavers, radiofrequency-probe and technique used for repair will be done or used according to the standard protocol at the site. During arthroscopy some of the degenerative tissue will be collected which would otherwise be removed during debridement. This includes tissue from the supraspinatus muscle, footprint and $2-3 \mathrm{~cm}$ of the insertion from the LHBT on the glenoid. The obtained tissue will be stained in paraffin and used for future research. The use of preoperative antibiotics and postoperative rehabilitation protocols will be standardised. The rehabilitation protocol consists of immobilisation using an anti-rotation sling directly after surgery and patients were instructed to start with pendulum exercises. From the first week after surgery, patients started physiotherapy with restricted passive range of motion up to $70^{\circ}$ of abduction, $70^{\circ}$ of forward elevation and $20^{\circ}$ of external rotation in the scapular plane. From 6 weeks, the immobilisation was phased out and (guided) active motion exercises were started. From 3 months on, active motion exercises above the shoulder level were allowed. The use of analgesics is allowed during the study. Patients will be asked for the use of analgesics during the study.

\section{Group 1: biceps tenotomy with cuff repair}

A biceps tenotomy with an additional cuff repair is performed. After surgery patients will be immobilised for 6 weeks in an anti-rotation sling. Two weeks after surgery the patients will visit the outpatient department for wound inspection and removal of stitches. After 6 and 12 weeks they will be checked on function and persistence of symptoms. Patients will be referred to a physiotherapist immediately after discharge of the hospital to assist in the passive mobilisation of the shoulder during the first 6 weeks, and the active mobilisation after 6 weeks.

\section{Group 2: biceps tenotomy without cuff repair}

An isolated biceps tenotomy is performed. After surgery shoulders are directly functional without any restrictions. A simple sling can be given to support the arm during the recovery period from surgery.

\section{Study outcomes}

The study outcomes at the different follow-up moments are shown in table 1 .

\section{Primary outcome}

The primary outcome is quality-of-life measured by the Western Ontario Rotator Cuff (WORC) Index 6 months after surgery. The WORC index is a disease-specific shoulder questionnaire, originally developed at the University of Western Ontario and has been validated in 
Table 1 Evaluation schedule

\begin{tabular}{llllllll}
\hline & & & $\mathbf{1 2}$ & $\mathbf{6}$ & & & \\
& Baseline & $\mathbf{6}$ weeks & weeks & months & 1 year & $\mathbf{2}$ years & $\mathbf{5}$ years \\
\hline WORC & $\mathrm{X}$ & $\mathrm{X}$ & $\mathrm{X}$ & $\mathrm{X}$ & $\mathrm{X}$ & $\mathrm{X}$ & $\mathrm{X}$ \\
Pain score & $\mathrm{X}$ & $\mathrm{X}$ & $\mathrm{X}$ & $\mathrm{X}$ & $\mathrm{X}$ & $\mathrm{X}$ & $\mathrm{X}$ \\
Constant-Murley score & $\mathrm{X}$ & & $\mathrm{X}$ & $\mathrm{X}$ & $\mathrm{X}$ & & \\
Passive glenohumeral motion & $\mathrm{X}$ & & $\mathrm{X}$ & $\mathrm{X}$ & $\mathrm{X}$ & & \\
Subjective shoulder value (SSV) & $\mathrm{X}$ & $\mathrm{X}$ & $\mathrm{X}$ & $\mathrm{X}$ & $\mathrm{X}$ & $\mathrm{X}$ & $\mathrm{X}$ \\
EQ-5D-5L & $\mathrm{X}$ & $\mathrm{X}$ & $\mathrm{X}$ & $\mathrm{X}$ & $\mathrm{X}$ & $\mathrm{X}$ & $\mathrm{X}$ \\
Hospital Anxiety and Depression Scale (HADS) & $\mathrm{X}$ & & & & $\mathrm{X}$ & & \\
Pain Catastrophyzing Scale (PCS) & $\mathrm{X}$ & & & & $\mathrm{X}$ & & \\
MRI & $\mathrm{X}$ & & & & & & \\
Ultrasound & & & & $\mathrm{X}$ & & & \\
Radiograph & $\mathrm{X}$ & & & & & & \\
Expectations/level of satisfactory & $\mathrm{X}$ & & $\mathrm{X}$ & $\mathrm{X}$ & $\mathrm{X}$ & $\mathrm{X}$ \\
Cost effectiveness & & & $\mathrm{X}$ & & $\mathrm{X}$ \\
\hline
\end{tabular}

EQ-5D-5L, EuroQol 5 Dimension 5 Level; WORC, Western Ontario Rotator Cuff.

Dutch. ${ }^{26}$ The SE of measurement for the WORC is 6.0 points. ${ }^{26}$ For this study, a difference of more than 10 points in WORC $0-100$ score between groups is considered clinically relevant.

\section{Secondary outcomes}

The secondary outcomes are:

- Difference in:

- Quality-of-life, measured by EuroQol 5 Dimension 5 Level(EQ-5D-5L) questionnaire. ${ }^{27}$

- Presence of anxiety and depression, measured by Hospital Anxiety and Depression Scale. ${ }^{28}$

- Pain Catastrophyzing Scale. ${ }^{29}$

- Pain during rest and during activity, measured on an 11-point Numeric Rating Scale.

- Subjective shoulder value.

- Function short term ( $\leq 6$ months) and long term ( $>6$ months to 1 year), measured by the ConstantMurley score and glenohumeral range of motion.

- Re-tear rate at 6 months determined by ultrasound in the repair group. Recurrent tears were defined as a distinct hypoechoic or mixed hyperechoic and hypoechoic defect in both transverse and longitudinal planes or when compression on the deltoid muscle with the probe could separate the torn tendon ends. ${ }^{30}$

- Cost effectiveness measured by Treatment Inventory of Costs in Patients (TiC-P part II). ${ }^{31}$

- Relation between participants functional change, their expectation and their satisfaction of treatment.

- Complication rate and adverse events.

- Interobserver and intraobserver reliability on reparability of stage 2-3 fatty degenerative rotator cuff tears among orthopaedic shoulder surgeons.
- Histological assessment of residual tissue derived from the rotator cuff tendons, biceps tendon and footprint (only in the main centre). The histological findings will among others be used for classifying rotator cuff tears in combination with imaging and perioperative findings.

At baseline a radiograph in antero-posterior and outlet view will be made. The presence of calcifications, stage of arthropathy, presence of arthrosis in the acromioclavicular joint, type acromion and AHI are scored. ${ }^{9}$ All MRIs will be systematically checked on the location of the tear in the sagittal plane, the amount of retraction of the supraspinatus tendon in the coronal plane, the amount of atrophy, pathology of the biceps tendon, acromiohumeral distance and presence of acromioclavicular arthrosis. ${ }^{52} 33$ All data management will be recorded online, unless patients are unable or unwilling to complete the questionnaires online. In that case, patients are asked to complete the questionnaires on paper.

\section{Sample size}

Sample size calculations (continuous outcome noninferiority trial) were performed using sealed envelope. ${ }^{34}$ The sample size was based on a power of $80 \%$, an alpha of 0.05 , an SD of 25 points and a non-inferiority limit (d) of 10 points on the mean WORC $(0-100)$ index. This data was obtained from previous studies using the WORC index. ${ }^{26}{ }^{35}$ We calculated that with $10 \%$ loss to follow-up, 86 patients were needed per group. This corresponds with a total of 172 patients that need to be included. Patients in both groups will be excluded per-operatively, when a cuff repair seems technically not possible, partial repair is performed or the biceps tendon is unimpaired. These 
patients will be monitored according to this protocol, however, these patients do not count towards the total number of patients. Inclusion finishes when both groups consist of 86 eligible patients.

\section{Data analysis}

All statistical analysis will be performed using SPSS version 24.0 or newer (SPSS). The Kolmogorov-Smirnov test will be used to determine whether continuous variables are normally distributed. Skewed data will be presented as median (range), normally distributed data will be presented as means (SD). For all analyses, a two-tailed value of $p \leq 0.05$ is considered to be significant.

To study the effect of the surgical interventions, the values from the baseline, and all follow-up moments in the tenotomy group will be compared with the effect in the group undergoing an additional cuff repair. To investigate the effect of the two surgical interventions, generalised estimating equations (GEE) for longitudinal analysis will be used. This method takes into account the dependency of observations within a patient and the fact that not all patients may be assessed at each time point (missing data). In the primary GEE model, the outcome variable studied (eg, WORC index) will be analysed as a dependent variable, using treatment allocation (1, tenotomy; 0 , tenotomy combined with cuff repair) and time as independent variables. In the secondary GEE model, the outcome variables studied (eg, Constant-Murley score, glenohumeral range of motion, pain score, ultrasound outcome (healed or non-healed cuff repair), EQ-5D-5L, patient's expectation and satisfaction and adverse events) will be analysed in a similar way. To evaluate whether the two groups differed in change over time, the interaction term of group and time (group * time) will be assessed. All models will be corrected for hospital of inclusion.

Both intention-to-treat and per-protocol analysis will be performed. The intention-to-treat approach will be the main analysis and provides unbiased comparisons among the treatment groups (avoid the effects of crossover and dropout, which may break the random assignment to the treatment groups). Patients who are excluded perioperatively will be excluded and replaced to prevent the risk of bias in allocation concealment.

Cohen's weighted kappa ( $\kappa$; ordinal categories) will be used to calculate interobserver and intraobserver agreement of the two raters of the MRI based variables (stage Goutallier fatty infiltration, tear size (Thomazeau A-E), retraction (Thomazeau 1-4), atrophy (Thomazeau 1-3), pathology of the biceps tendon (Chen 1-6), acromiohumeral distance and presence of acromioclavicular arthrosis. A kappa of 0 represents agreement equivalent with random change alone, whereas a kappa of 1 represents perfect agreement.

Using the histological assessment, imaging and perioperative findings, the rotator cuff tears will be classified. The impact of this classification, the MRI based variables and other patient-related variables (eg, gender, age) as prognostic factors on the dependent variables WORC,
Constant-Murley score and pain will be evaluated using regression analysis.

\section{Cost-effectiveness analysis}

The cost-effectiveness analysis will be conducted from a societal perspective. To establish the costs, relevant cost items are identified, after which these costs are measured and valued. These relevant cost items are: the total surgery time, use of surgical devices (eg, anchors, cannula's, sutures) and length of stay in the hospital. In addition, medication use, physiotherapy, general practitioner care, costs of visits to other primary care providers, ambulatory and inpatient hospital care are taken into account. By dividing the difference in mean total costs between the groups by the difference in mean effects the incremental cost-effectiveness ratios will be calculated. The time horizon of the economic evaluation is 12 months. Utility values will be calculated for the health states of the EQ-5D-5L based on the Dutch tariff for the EuroQol. ${ }^{27} 37$ The utility values will be used to compute quality-adjusted life-years (QALY-EQ-5D-5L) by means of the area under the curve method. TiC-P part II is used to measure costs and consequences of productivity losses (indirect costs). ${ }^{31}$ It has four modules: absenteeism from paid work, production losses without absenteeism from paid work, unpaid work and nuisance in paid and unpaid work and contains of 11 questions. A 'budget impact analysis' will be performed from societal, government and insurer perspective. Resource utilisation is calculated by multiplying the number of eligible patients with the resource utilisation rates obtained from the cost-effectiveness analysis. $^{38} 39$

We expect that for all relevant cost categories the costs of an isolated biceps tenotomy are lower than those of the biceps tenotomy with cuff repair. We expect that direct healthcare related costs are lower, because surgery for patients undergoing an isolated biceps tenotomy will be shorter and less surgical instruments and materials are needed. We expect that direct non-healthcare related costs are lower, because we hypothesise that the complication rate is lower, which leads to fewer hospital visits and thus less travel time. We expect that indirect healthcare related costs are absent, because both biceps tenotomy treatments do not affect life expectancy. We expect that indirect non-healthcare related costs are lower, because patients undergoing an isolated biceps tenotomy treatment resume work much faster, which leads to lower productivity losses. We expect that an isolated biceps tenotomy treatment leads to increased patient utility.

\section{Handling and storage of data}

Data will be collected using a web based electronic data collection system (REDCap). REDCap has been designed to ensure the security of patient health information and allows for direct entry by the subject, investigator and study coordinator. ${ }^{25}$ Precautions are in place to secure the data and prevent unauthorised access, use, and information disclosure, including use of web-based passwords 
and encryption. All subject data will be anonymised by assigning study numbers to each subject. The key to these study numbers is only available to the coordinating investigator and the principal investigators. Outcome data, anonymised, is only accessible for the coordinating investigator, principal investigators and statistical analysers. Data will be processed and stored in SPSS which will be password protected. The handling of personal data will comply with the General Data Protection Regulation. Data will be collected and stored for a period of 15 years. Signed informed consent documents, paper copies with patient names and unique identifiers as well as paper questionnaires will be stored at the centre of inclusion. Documents will be maintained in a locked cabinet outside the medical record area. Digital data will be maintained on password protected, secure computers.

\section{ETHICS AND DISSEMINATION}

The results of this study will be reported in peer-reviewed journals and at (inter)national conferences. Furthermore, we will share our findings with the appropriate guideline committees.

\section{PATIENT AND PUBLIC INVOLVEMENT}

This study has been developed because we noticed that patients experienced the rehabilitation after cuff repair as difficult and they struggle with the fact that rehabilitation takes so long. As we read in literature and experienced excellent results of the isolated tenotomy of the biceps tendon in the same patient group, we decided to start this clinical trial. With the exception of the emergence of the research question, patients were not involved in the design or set-up of the study. The burden of the study was not assessed before commencement. After finishing the study, patients will be informed about the general results of the study using a pamphlet.

Contributors All authors have contributed to the design of this trial protocol. FH, NW and GAY have contributed to writing the protocol and this manuscript. GAY is the principal investigator of this trial. All authors have contributed to the manuscript and read and approved the final manuscript.

Funding This work was supported by the foundation of medical research of the St. Antonius Hospital grant number [14.8]. The foundation was not and will not be involved in the design, data collection, analysis, interpretation, writing or any other aspects of the study.

Competing interests None declared.

Patient consent for publication Not required.

Ethics approval The Medical Research Ethics Committees United (MEC-U), Nieuwegein, the Netherlands gave approval for this trial; registration number NL54313.100.15. The start date (inclusion of the first patient) was 22 0ctober 2018. Furthermore, all institutional review boards of the participating centres gave approval. The study will be performed in accordance with the Declaration of Helsinki with the Medical Research Involving Human Subjects Act (WMO). All adverse events, other than pain, muscle cramp and loss of strength which are normal findings after these treatments, reported by the subject or observed by the investigator or his staff will be recorded. All SAEs will be reported to the accredited ethics committee that approved the protocol. We will inform the subjects and the reviewing accredited ethics committee if anything occurs, on the basis of which it appears that the disadvantages of participation may be significantly greater than was foreseen in the research proposal. The study will be suspended pending further review by the accredited ethics committee, except insofar as suspension would jeopardise the subjects' health. The investigator will take care that all subjects are kept informed. All substantial amendments to the. study protocol will be notified to the Ethics Committee that gave a favourable opinion.

Provenance and peer review Not commissioned; externally peer reviewed.

Open access This is an open access article distributed in accordance with the Creative Commons Attribution Non Commercial (CC BY-NC 4.0) license, which permits others to distribute, remix, adapt, build upon this work non-commercially, and license their derivative works on different terms, provided the original work is properly cited, appropriate credit is given, any changes made indicated, and the use is non-commercial. See: http://creativecommons.org/licenses/by-nc/4.0/.

ORCID iD

Nienke Wolterbeek http://orcid.org/0000-0003-1745-1417

\section{REFERENCES}

1 Milgrom C, Schaffler M, Gilbert S, et al. Rotator-Cuff changes in asymptomatic adults. The effect of age, hand dominance and gender. J Bone Joint Surg Br 1995;77-B:296-8.

2 Sher JS, Uribe JW, Posada A, et al. Abnormal findings on magnetic resonance images of asymptomatic shoulders. J Bone Joint Surg Am 1995; 77:10-15.

3 Yamaguchi K, Ditsios K, Middleton WD, et al. The demographic and morphological features of rotator cuff disease. A comparison of asymptomatic and symptomatic shoulders. J Bone Joint Surg Am 2006;88:1699-704.

4 Goutallier D, Postel JM, Bernageau J, et al. Fatty muscle degeneration in cuff ruptures. pre- and postoperative evaluation by CT scan. Clin Orthop Relat Res 1994:78-83.

5 Thomazeau H, Boukobza E, Morcet N, et al. Prediction of rotator cuff repair results by magnetic resonance imaging. Clin Orthop Relat Res 1997;344:275-83.

6 Boileau P, Baqué F, Valerio L, et al. Isolated arthroscopic biceps tenotomy or tenodesis improves symptoms in patients with massive irreparable rotator cuff tears. J Bone Joint Surg Am 2007;89:747-57.

7 Walch G, Edwards TB, Boulahia A, et al. Arthroscopic tenotomy of the long head of the biceps in the treatment of rotator cuff tears: clinical and radiographic results of 307 cases. J Shoulder Elbow Surg 2005;14:238-46.

8 Maynou C, Mehdi N, Cassagnaud X, et al. [Clinical results of arthroscopic tenotomy of the long head of the biceps brachii in full thickness tears of the rotator cuff without repair: 40 cases]. Rev Chir Orthop Reparatrice Appar Mot 2005;91:300-6.

9 Hamada K, Fukuda H, Mikasa M, et al. Roentgenographic findings in massive rotator cuff tears. A long-term observation. Clin Orthop Relat Res 1990:92-6.

10 Burkhart SS, Barth JRH, Richards DP, et al. Arthroscopic repair of massive rotator cuff tears with stage 3 and 4 fatty degeneration. Arthroscopy 2007;23:347-54.

$11 \mathrm{Kim} \mathrm{KC}$, Shin HD, Lee WY, et al. Repair integrity and functional outcome after arthroscopic rotator cuff repair: double-row versus suture-bridge technique. Am J Sports Med 2012;40:294-9.

12 Meyer DC, Wieser K, Farshad M, et al. Retraction of supraspinatus muscle and tendon as predictors of success of rotator cuff repair. Am J Sports Med 2012;40:2242-7.

13 Chung SW, Kim JY, Kim MH, et al. Arthroscopic repair of massive rotator cuff tears: outcome and analysis of factors associated with healing failure or poor postoperative function. Am J Sports Med 2013;41:1674-83.

14 Arrigoni P, Fossati C, Zottarelli L, et al. Functional repair in massive immobile rotator cuff tears leads to satisfactory quality of living: results at 3-year follow-up. Musculoskelet Surg 2013;97:73-7.

15 Park J-Y, Lhee S-H, Oh K-S, et al. Clinical and ultrasonographic outcomes of arthroscopic suture bridge repair for massive rotator cuff tear. Arthroscopy 2013;29:280-9.

16 Cho NS, Rhee YG. The factors affecting the clinical outcome and integrity of arthroscopically repaired rotator cuff tears of the shoulder. Clin Orthop Surg 2009;1:96-104.

17 Shen $\mathrm{P}-\mathrm{H}$, Lien S-B, Shen $\mathrm{H}-\mathrm{C}$, et al. Long-Term functional outcomes after repair of rotator cuff tears correlated with atrophy of the supraspinatus muscles on magnetic resonance images. J Shoulder Elbow Surg 2008;17:S1-7.

18 Pill SG, Walch G, Hawkins RJ, et al. The role of the biceps tendon in massive rotator cuff tears. Instr Course Lect 2012;61:113-20. 
19 De Carli A, Vadalà A, Zanzotto E, et al. Reparable rotator cuff tears with concomitant long-head biceps lesions: tenotomy or tenotomy/ tenodesis? Knee Surg Sports Traumatol Arthrosc 2012;20:2553-8.

20 Dezaly C, Sirveaux F, Philippe R, et al. Arthroscopic treatment of rotator cuff tear in the over-60s: repair is preferable to isolated acromioplasty-tenotomy in the short term. Orthop Traumatol Surg Res 2011;97:S125-30.

$21 \mathrm{Koh} \mathrm{KH}$, Ahn JH, Kim SM, et al. Treatment of biceps tendon lesions in the setting of rotator cuff tears: prospective cohort study of tenotomy versus tenodesis. Am J Sports Med 2010;38:1584-90.

22 Pavlidis T, Ganten M, Lehner B, et al. [Tenoplasty of the long head of the biceps in massive rotator cuff tear]. Z Orthop Ihre Grenzgeb 2003;141:177-81.

23 Checchia SL, Doneux PS, Miyazaki AN, et al. Biceps tenodesis associated with arthroscopic repair of rotator cuff tears. J Shoulder Elbow Surg 2005;14:138-44.

24 Uzel A-P, Bertino R, Boileau P. Bilateral spontaneous tenodesis of the long head of the biceps at the bicipital groove with massive rotator cuff tear on a 103-year-old female cadaver: the natural evolution of the human shoulder? Musculoskelet Surg 2011;95:79-82.

25 Harris PA, Taylor R, Thielke R, et al. Research electronic data capture (REDCap) - A metadata-driven methodology and workflow process for providing translational research informatics support. J Biomed Inform 2009;42:377-81.

26 Wessel RN, Wolterbeek N, Fermont AJM, et al. The conceptually equivalent Dutch version of the Western Ontario Rotator Cuff Index (WORC)@. BMC Musculoskelet Disord 2013;14:362-8.

27 EuroQol Group. EuroQol - a new facility for the measurement of health-related quality of life. Health Policy 1990;16:199-208.

28 Spinhoven PH, Ormel J, Sloekers PPA, et al. A validation study of the hospital anxiety and depression scale (HADS) in different groups of Dutch subjects. Psychol Med 1997;27:363-70.

29 Osman A, Barrios FX, Kopper BA, et al. Factor structure, reliability, and validity of the pain Catastrophizing scale. J Behav Med 1997;20:589-605.
30 Hakkaart-van Roijen L. Handleiding TiC-P (vragenlijst voor zorggebruik en productieverliezen bij psychische aandoeningen. Rotterdam: iMTA, Erasmus Universiteit Rotterdam, 2010.

31 Hollman F, Wolterbeek N, Zijl JAC, et al. Abduction brace versus Antirotation sling after arthroscopic cuff repair: the effects on pain and function. Arthroscopy 2017;33:1618-26.

32 Chen C-H, Chang C-H, Su C-I, et al. Classification and analysis of pathology of the long head of the biceps tendon in complete rotator cuff tears. Biomed J 2012;35:263-70.

33 de Abreu MR, Chung CB, Wesselly M, et al. Acromioclavicular joint osteoarthritis: comparison of findings derived from MR imaging and conventional radiography. Clin Imaging 2005;29:273-7.

34 Sealed Envelope Ltd. Power calculator for continuous outcome non-inferiority trial. [Online], 2012. Available: https://www. sealedenvelope.com/power/continuous-noninferior/ [Accessed Apr 2019].

35 Björnsson HC, Norlin R, Johansson K, et al. The influence of age, delay of repair, and tendon involvement in acute rotator cuff tears: structural and clinical outcomes after repair of 42 shoulders. Acta Orthop 2011;82:187-92

36 MacDonald P, McRae S, Leiter J, et al. Arthroscopic rotator cuff repair with and without acromioplasty in the treatment of fullthickness rotator cuff tears: a multicenter, randomized controlled trial. J Bone Joint Surg Am 2011;93:1953-60.

37 Lamers LM, Stalmeier PFM, McDonnell J, et al. [Measuring the quality of life in economic evaluations: the Dutch EQ-5D tariff]. Ned Tijdschr Geneeskd 2005;149:1574-8.

38 Stinnett AA, Mullahy J. Net health benefits: a new framework for the analysis of uncertainty in cost-effectiveness analysis. Med Decis Making 1998;18:S68-80.

39 Fenwick E, O'Brien BJ, Briggs A. Cost-effectiveness acceptability curves--facts, fallacies and frequently asked questions. Health Econ 2004;13:405-15. 